\title{
Reduced lung function and risk of atrial fibrillation in The Copenhagen City Heart Study
}

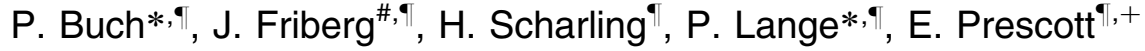

Reduced lung function and risk of atrial fibrillation in The Copenhagen City Heart Study. P. Buch, J. Friberg, H. Scharling, P. Lange, E. Prescott. (C) ERS Journals Ltd 2003. ABSTRACT: Chronic obstructive pulmonary disease has been associated with a high frequency of arrhythmias. Few studies have analysed the role of reduced lung function in predicting atrial fibrillation (AF). The aim of the present study was to investigate the relationship between forced expiratory volume in one second (FEV1) and risk of first episode of AF in a prospective study.

Data from 13,430 males and females without previous myocardial infarction, who participated in the Copenhagen City Heart Study, were analysed. New AF was assessed at re-examination after 5 yrs and by hospital admission for AF during a period of 13 yrs. Multivariate analyses were used with adjustment for cardiopulmonary risk factors. There were 62 new cases of $\mathrm{AF}$ at 5-yr follow-up $(0.58 \%)$ and 290 cases $(2.20 \%)$ diagnosed at hospitalisations.

Risk of new AF at re-examination was 1.8-times higher for FEV1 between $60-80 \%$ of predicted compared with FEV1 $\geqslant 80 \%$ after adjustment for sex, age, smoking, blood pressure, diabetes and body mass index. The risk of AF hospitalisation was 1.3-times higher for FEV1 between $60-80 \%$ and 1.8-times higher for FEV1 $<60 \%$ compared with FEV1 $\geqslant 80 \%$, when additional adjustment was made for education, treatment with diuretics and chest pain at activity.

The authors conclude that reduced lung function is an independent predictor for incident atrial fibrillation.

Eur Respir J 2003; 21: 1012-1016.
*Dept of Respiratory Medicine, Hvidovre University Hospital, ${ }^{\#}$ Dept of Cardiovascular Medicine, Bispebjerg University Hospital, ${ }^{\top}$ The Copenhagen City Heart Study, Bispebjerg University Hospital, and ${ }^{+}$Institute of Preventive Medicine, Kommunehospitalet, Copenhagen, Denmark.

Correspondence: P. Buch, Dybboelsgade 14, 3. tv., DK-1721 Copenhagen, Denmark.

Fax: 4539751803

E-mail: pbuch@dadlnet.dk

Keywords: Atrial fibrillation, epidemiology, follow-up studies, forced expiratory volume in one second, risk factors

Received: June 162002

Accepted after revision: January 92003

The Copenhagen City Heart Study was funded by the Danish Heart Foundation. P. Buch was the recipient of a Danish Heart Foundation introduction grant (01-1-9-F3-22880).
Chronic obstructive pulmonary disease (COPD) has been associated with a high frequency of cardiac arrhythmias. Hypoxaemia [1, 2], acidosis [3], cor pulmonale and coexisting ischaemic heart disease (IHD) $[4,5]$ have been proposed as major causes for the relationship between COPD and arrhythmias. The risk of arrhythmias in patients with COPD is influenced by the state of the disease, with a higher frequency of supraventricular tachycardia during exacerbations [1, 2, 5]. However, even in patients with stable COPD the incidence of cardiac arrhythmias is considerable [6], and, in clinical practice, it is not uncommon to find atrial fibrillation (AF) in patients hospitalised for COPD. AF is by far the most common arrhythmia in the elderly population but only a few studies have analysed the relationship between lung function and the risk of developing AF in detail. It is important to investigate the correlation between reduced lung function and AF since the incidence of COPD is expected to increase considerably in the future, reflecting prior smoking habits of an ageing population [7, 8]. The aim of the present study, therefore, was to investigate the role of reduced lung function in development of AF based on a representative sample of the general population free of IHD at baseline.

\section{Methods}

\section{Study population}

This study was based on data from the Copenhagen City Heart Study (CCHS). The original cohort of CCHS comprised a random age-stratified sample of 19,329 males and females aged $\geqslant 20$ yrs from an area of Copenhagen, Denmark. A total of 14,223 persons participated in the first examination between 1976-1978. Re-examination took place after 5 yrs (1981-1983). The overall response rates were of $74 \%$ and $70 \%$, respectively. At each examination, cardiovascular and respiratory risk factors were obtained by a detailed self-administered questionnaire and several laboratory tests. Details of the examination procedure have been described previously [9]. Since the aim of this study was to investigate lung function as a predictor of AF, only baseline data from the first examination were used. Subjects with pre-existing AF ( $n=87)$ were excluded from further analyses. To diminish contribution of pre-existing IHD, an additional 386 subjects with prior myocardial infarction (MI) were excluded. Among the remaining participants, 25 subjects with extreme values of forced expiratory volume in one second (FEV1) \% predicted lower than $10 \%$ or higher than $140 \%$, and subjects with missing FEV1 \% pred or electrocardiograms (ECGs; n=295) were excluded. Thus, a total of 13,430 subjects $(6,084$ males and 7,346 females) were included at baseline.

At 5-yr follow-up, 10,670 subjects $(79 \%)$ were re-examined and were eligible for analyses of new AF detected at examination. Cox regression analyses of hospitalisations with AF were restricted to 13,181 subjects because of lack of one or more covariates at baseline $(n=249)$. Between the first and second examination, 756 subjects died. Of those who were eligible for hospitalisation analyses a total of 2,956 subjects had died at the end of follow-up (December 31, 1990). Compared with responders at re-examination, nonresponders 
had lower FEV1 \% pred, higher systolic blood pressure, higher nonfasting blood sugar, more frequent diabetes and were more often current smokers, all measured at baseline. There was no difference in age and body mass index (BMI). Thus, nonresponders in general had a poorer cardiopulmonary risk profile than responders.

\section{New cases of atrial fibrillation}

Two outcomes of occurrence of AF were used: 1) presence of AF after 5-yr follow-up at re-examination; and 2) hospital admission with a diagnosis of AF until December 31, 1990. A 12-lead resting ECG was recorded using a Mingograf 34 (Elema-Siemens, Solna, Sweden) at both health examinations. All ECGs were coded independently by two laboratory technicians according to the Minnesota coding system and in case of disagreement a third coder settled the disagreement [10]. No distinction was made between AF and atrial flutter. A random sample of $10 \%$ of the population $>65$ yrs of age was selected and ECGs were reviewed by a physician who also made reviews of all ECGs originally coded as AF. The random sample revealed two subjects $(1.0 \%)$ at baseline who were misclassified as not having AF. No misclassifications were found at 5-yr follow-up. At review, all ECGs originally coded as AF were found to be consistent at baseline, whereas three subjects $(2.7 \%)$ at 5-yr follow-up did not have AF.

Hospital discharge diagnoses were obtained from the National Hospital Discharge Register and International Classification of Diseases (ICD) codes for new AF or atrial flutter in any coding position were identified (ICD-8 codes 427.93 or 427.94). As AF in subjects with, for example, COPD or IHD leading to hospital admission is more likely to be diagnosed than in subjects with lone AF, this might cause bias [11]. Separate analyses using only hospital admission with $\mathrm{AF}$ as the main diagnosis (first code) were therefore performed. Furthermore, the primary cause (main diagnosis) for hospital admission for those subjects who had AF diagnosed in the second or higher coding position, according to the National Hospital Discharge Register, was obtained. All analyses were based on the first ever hospitalisation with AF for participants without AF or IHD at baseline. Followup was limited to 13 yrs (December 31, 1990), since it was assumed that exposure measured at baseline would be too imprecise compared with the end-point if a longer follow-up period was chosen.

\section{Pulmonary function tests}

FEV1 and forced vital capacity (FVC) were measured at baseline using an electronic spirometer (Monaghan N 403; Monaghan, Littleton, CO, USA), which had measurement accuracy within $5 \%$ of volume. The spirometer was calibrated daily with a $1-\mathrm{L}$ syringe and weekly against a water-sealed Godard spirometer (Sensormedics, Bilthoven, the Netherlands). Specially trained technicians performed all measurements. Three forced expiratory manoeuvres were obtained and, as a criterion for correct performance of the procedure, at least two measurements differing $<5 \%$ had to be produced. The highest value of FEV1 and FVC were used in the analyses. FEV1 \% pred was calculated separately for males and females using coefficients obtained from linear regressions, based on a subgroup of healthy never-smokers [12]. The following formulae for males (Equation 1) and females (Equation 2) were used:

$$
\mathrm{FEV}_{1}(\mathrm{~mL})=-506-38 \times \text { age }(\mathrm{yrs})+35 \times \text { height }(\mathrm{cm})
$$

$$
\mathrm{FEV}_{1}(\mathrm{~mL})=443-30 \times \text { age }(\mathrm{yrs})+23 \times \text { height }(\mathrm{cm})
$$

FEV1 \% pred was categorised into three subgroups: $<60 \%$, $60-80 \%$ and $\geqslant 80 \%$.

\section{Confounders}

Among the available data on the CCHS participants, only confounders that were shown to be independent risk factors of AF in earlier studies were chosen [13-17]. Thus, corrections were made for hypertension, IHD, BMI, diabetes, age and sex. Valvular heart disease and congestive heart failure have also been shown to be strong predictors of AF, but no information was available. As a surrogate parameter for congestive heart failure, treatment with diuretics was used. Tobacco smoking and levels of education have not been shown to be predictors but were included since they could influence FEV1. Arterial blood pressure was measured in a sedentary position after 5-min rest. Systolic blood pressure was divided into three categories: <140, 140-159 and $\geqslant 160 \mathrm{mmHg}$. Previous MI was defined as self-reported MI, verified by hospital records or a personal general practitioner, or as pathological Q-waves detected on ECGs at baseline. Possible angina was defined as self-reported chest pain at activity. BMI was calculated as weight $(\mathrm{kg})$ divided by height squared $\left(\mathrm{m}^{2}\right)$ and divided into three categories: $<25,25-29$ and $\geqslant 30 \mathrm{~kg} \cdot \mathrm{m}^{-2}$. Diabetes was considered present if nonfasting blood glucose was $\geqslant 11.1 \mathrm{mM}\left(200 \mathrm{mg} \cdot \mathrm{dL}^{-1}\right)$ or the subjects answered "yes" to the question: "Do you have diabetes?" Tobacco smoking was studied in five categories: neversmokers, exsmokers and current smokers of 1-10, 11-24 and $\geqslant 25 \mathrm{~g}$ tobacco per day. Type of tobacco (cigarette, cheroot, cigar, pipe or mixed) was recorded for current smokers, and tobacco consumption was calculated by equating a cigarette to $1 \mathrm{~g}$, a cheroot to $3 \mathrm{~g}$, and a cigar to $5 \mathrm{~g}$ of tobacco. Level of education was divided into three groups: $<9,9-11$ and $\geqslant 12$ yrs of school.

\section{Statistics}

One-way variance analysis was used to compare continuous variables between groups. The Chi-squared test was used for categorical variables. Logistic regression was used to identify independent predictors of new AF at re-examination. First, each variable of interest was tested, adjusting only for age (by $10-y r$ age groups) and sex. The final model was tested for interaction between sex and each covariate.

Association between risk factors and AF detected by hospital discharge diagnosis was analysed using Cox proportional hazards regression models [18] with the first hospitalisation for AF as end-point. Age and delayed entry were used as the underlying time axes with subjects entering analyses at their age of study inclusion. Subjects who experienced an event (AF) only contributed with observation time until the time of this event. Those who died before the end of follow-up contributed with observation time until the time of their death at which time they were censored. Relative risk (RR) for FEV1 \% pred did not differ between males and females and the final analyses were performed on the whole sample stratified by sex. The limit of significance was $5 \%$ for all analysis.

\section{Results}

\section{Baseline characteristics}

Table 1 shows the correlation between level of lung function and selected characteristics of the population at 
Table 1.-Baseline characteristics of the study population without pre-existing atrial fibrillation in accordance with the level of forced expiratory volume in one second (FEV 1 ) \% predicted

\begin{tabular}{|c|c|c|c|}
\hline \multirow[t]{2}{*}{ Characteristics } & \multicolumn{3}{|c|}{ FEV1 \% pred } \\
\hline & $<60 \%$ & $60-80 \%$ & $\geqslant 80 \%$ \\
\hline Subjects $n$ & 1238 & 3872 & 8320 \\
\hline Females & $588(47.5)$ & $2065(53.3)$ & $4693(56.4)$ \\
\hline Age yrs & $56.5 \pm 10.2$ & $52.6 \pm 11.8$ & $51.4 \pm 12.1$ \\
\hline $\mathrm{BMI} \mathrm{kg} \cdot \mathrm{m}^{-2}$ & $25.4 \pm 4.9$ & $25.4 \pm 4.4$ & $25.0 \pm 3.9$ \\
\hline Systolic blood pressure $\mathrm{mmHg}$ & $141.3 \pm 23.0$ & $138.1 \pm 21.9$ & $135.8 \pm 21.2$ \\
\hline Diastolic blood pressure $\mathrm{mmHg}$ & $84.9 \pm 12.8$ & $82.9 \pm 12.2$ & $82.4 \pm 12.2$ \\
\hline Blood glucose $\mathrm{mm} \cdot \mathrm{L}^{-1}$ & $6.82 \pm 2.06$ & $6.73 \pm 1.96$ & $6.52 \pm 1.50$ \\
\hline FEV1/FVC $\%$ & $63.9 \pm 13.8$ & $75.6 \pm 9.4$ & $82.7 \pm 7.6$ \\
\hline Current smokers & $904(73.0)$ & $2818(72.8)$ & $4827(58.0)$ \\
\hline Daily tobacco consumption $\mathrm{g}^{\#}$ & $12.0 \pm 10.6$ & $12.1 \pm 10.6$ & $9.0 \pm 10.4$ \\
\hline Exsmokers & $186(15.1)$ & $515(13.3)$ & $1517(18.3)$ \\
\hline Chest pain at activity & $317(25.6)$ & $566(14.6)$ & $739(8.9)$ \\
\hline Diabetes & $66(5.3)$ & $140(3.6)$ & $168(2.0)$ \\
\hline Treated with diuretics & $165(13.5)$ & $348(9.0)$ & $615(7.4)$ \\
\hline Education $<9$ yrs of school & $1003(81.3)$ & $2636(68.2)$ & $4833(58.2)$ \\
\hline
\end{tabular}

Data are presented as mean \pm SD or $\mathrm{n}(\%)$ unless otherwise indicated. BMI: body mass index. ${ }^{\#}$ : among current smokers. For all characteristics $\mathrm{p}<0.001$.

baseline. In general, there was an inverse relationship between FEV1 \% pred and biological, socioeconomical and cardiopulmonary risk factors. Thus, participants with lower levels of lung function were older, had higher systolic and diastolic blood pressure, higher mean blood glucose, higher BMI, lower mean $\mathrm{FEV1/FVC}$ and more frequent chest pain or diabetes. They also more often had $<9$ yrs of education and were current smokers. There was no difference in BMI or tobacco consumption between the lowest group of FEV1 and the middle group. When analyses were performed separately for males and females (data not shown), similar trends were seen.

\section{Risk of incident atrial fibrillation at 5-yr follow-up examination}

At the re-examination, 62 new cases of AF were registered. Table 2 shows the distribution of cases of AF at baseline, re-examination and at hospital admission. With lower levels of lung function, the prevalence of AF increased. This was seen both for AF at re-examination and for hospital admissions of AF.

Table 2. - Presence of atrial fibrillation (AF) at baseline, at re-examination and at incident hospitalisations according to lung function

\begin{tabular}{lccc}
\hline & \multicolumn{3}{c}{ FEV1 \% pred } \\
\cline { 2 - 4 } & $<60 \%$ & $60-80 \%$ & $\geqslant 80 \%$ \\
\hline At baseline & & & \\
$\quad$ Subjects n & 1253 & 3904 & 8351 \\
$\quad$ Presence of AF & $15(1.20)$ & $32(0.82)$ & $31(0.37)$ \\
At re-examination & & & \\
$\quad$ Subjects n & 809 & 2947 & 6910 \\
$\quad$ Presence of AF & $9(1.11)$ & $25(0.85)$ & $28(0.41)$ \\
At hospital admission & & & \\
$\quad$ Subjects n & 1167 & 3699 & 8315 \\
$\quad$ All cases of AF & $47(4.03)$ & $96(2.60)$ & $147(1.77)$ \\
AF as main diagnosis & $46(3.94)$ & $28(0.76)$ & $19(0.23)$ \\
\hline
\end{tabular}

Data are presented as n (\%) unless otherwise stated. FEV1\% pred: forced expiratory volume in one second $\%$ predicted.
There was an inverse relationship between FEV1 and risk of AF after 5 yrs in the model, adjusting for age and sex only (table 3). After full adjustment for all confounders the inverse association between FEV1 and AF was attenuated but remained significant. This association was only seen for FEV1 between $60-80 \%$ of predicted and reduction of FEV1 below $60 \%$ was not associated with AF in any of the models. Only age, sex, systolic blood pressure, diabetes and BMI were significantly associated with AF after multivariate adjustment.

In subanalyses, degrees of obstruction (FEV1/FVC) and risk of AF were investigated but no association was found in the age- and sex-adjusted model, and therefore this parameter was not included in the following analyses.

\section{Risk of incident atrial fibrillation at hospitalisations}

Hospital admissions recorded a total of 290 subjects with new AF. In $93(32.1 \%)$ cases, AF was mentioned as the main cause of hospital admission. In the remaining 187 cases, the most common main diagnoses were IHD (20.6\%), followed by MI $(14.3 \%)$, pneumonia $(9.1 \%)$, congestive heart failure $(5.7 \%)$, stroke $(3.4 \%)$ and COPD/asthma $(2.9 \%)$.

Figure 1 outlines the RRs of all hospitalisations with new AF for each $10 \%$ decline in FEV1\% pred, adjusted for age and

Table 3.-Reduced lung function and odds ratio (95\% confidence interval) of atrial fibrillation at re-examination after 5 yrs

\begin{tabular}{lcc}
\hline Variable & Model $1^{\#}$ & Model 2 \\
\hline Age $^{+}$ & $1.07(1.04-1.09)^{* * *}$ & $1.06(1.03-1.08)^{* * *}$ \\
Females & $1(1)$ & $1(1)$ \\
Males & $2.62(1.53-4.48)^{* * *}$ & $2.38(1.37-4.14)^{* *}$ \\
FEV1\% pred & $1(1)$ & $1(1)$ \\
$\quad \geqslant 80 \%$ & $1.94(1.13-3.34)^{*}$ & $1.75(1.01-3.05)^{*}$ \\
$60-80 \%$ & $2.03(0.95-4.34)$ & $1.64(0.74-3.65)$ \\
$<60 \%$ &
\end{tabular}

Data are presented as odds ratio (95\% confidence interval). FEV1 \% pred: forced expiratory volume in one second $\%$ predicted. ${ }^{\#}$ : adjusted only for sex and age; ": adjusted for sex, age, systolic blood pressure, diabetes and body mass index; ${ }^{+}$: per 10 -yr increase. *: $\mathrm{p}<0.05$; **: $\mathrm{p}<0.01 ; * * *: \mathrm{p}<0.001$. 


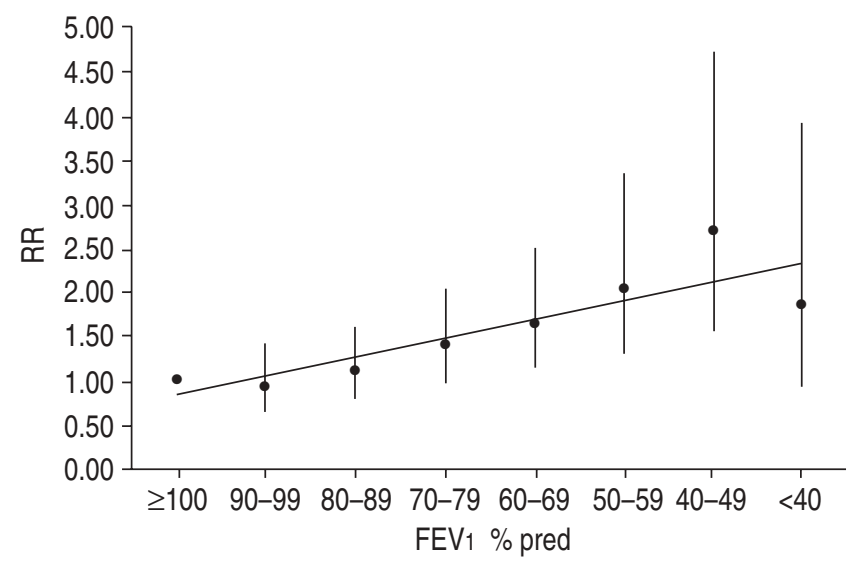

Fig. 1. - Forced expiratory volume in one second \% predicted (FEV1 $\%$ pred) and relative risk (RR) of hospital admission for new atrial fibrillation. Based on Cox regression model stratified by sex and age. Bars indicate the $95 \%$ confidence intervals. —: regression line.

sex. A trend towards a dose/response relationship between AF and reduced lung function was observed. The RRs were only slightly affected by adjustment for all selected confounders (table 4). The association remained similar, independent of whether AF was recorded as the main or as a contributory cause of admission.

\section{Discussion}

In the present prospective cohort study, the authors found reduced lung function to be significantly associated with new onset of AF measured as point prevalence after 5 yrs and as subsequent hospital admission with AF. The risk of AF with reduced lung function was unaffected by adjustment for several confounding factors. The risk for AF hospitalisation showed a trend towards a linear dose/response relationship with reduced lung function, except for the group with FEV1 $\%$ pred $<40 \%$ This could be partly explained by the small number of subjects with AF in this group $(n=23)$, making the estimate more imprecise as reflected in the wider confidence interval. In contrast to hospitalisations, the number of deaths in the group with the lowest level of lung function was considerably higher, i.e. $55(22 \%)$ subjects with FEV1 $<40 \%$ pred died during the 5-yr follow-up. Therefore the probability

Table 4. - Reduced lung function and relative risk (RR) (95\% confidence interval $(\mathrm{Cl})$ ) of incident atrial fibrillation (AF) hospitalisation

\begin{tabular}{|c|c|c|c|}
\hline \multirow{2}{*}{$\begin{array}{l}\text { New AF } \\
\text { hospitalisation }\end{array}$} & \multicolumn{2}{|r|}{ Model $1^{\#}$} & Model 2 \\
\hline & $\begin{array}{l}\text { FEV1 \% } \\
\text { pred }\end{array}$ & $\mathrm{RR}(95 \% \mathrm{CI})$ & $\mathrm{RR}(95 \% \mathrm{CI})$ \\
\hline \multirow{3}{*}{$\begin{array}{l}\text { All cases of } \\
\text { AF }\end{array}$} & $\geqslant 80 \%$ & $1(1)$ & $1(1)$ \\
\hline & $60-80 \%$ & $1.46(1.13-1.89)^{* *}$ & $1.31(1.00-1.70)^{*}$ \\
\hline & $<60 \%$ & $2.14(1.54-2.98)^{* * *}$ & $1.82(1.29-2.56)^{* *}$ \\
\hline \multirow{3}{*}{$\begin{array}{c}\mathrm{AF} \text { as main } \\
\text { diagnosis }\end{array}$} & $\geqslant 80 \%$ & $1(1)$ & $1(1)$ \\
\hline & $60-80 \%$ & $1.37(0.85-2.19)$ & $1.28(0.79-2.06)$ \\
\hline & $<60 \%$ & $2.83(1.65-4.85)^{* * *}$ & $2.53(1.45-4.42)^{* *}$ \\
\hline
\end{tabular}

Results are from Cox proportional hazards regression analysis. FEV1 \% pred: forced expiratory volume in one second \% predicted. ${ }^{\#}$ : stratified by sex, adjusted only for age; ${ }^{\uparrow}$ : stratified by sex and adjusted for age, systolic blood pressure, diabetes, treatment with diuretics, body mass index, chest pain, smoking and education. ${ }^{*}: \mathrm{p}<0.05 ; * *: \mathrm{p}<0.01 ; * * *$ : $\mathrm{p}<0.001$. of having AF diagnosed was limited, which could have contributed to the lower estimate.

The results from previous multivariate cohort studies are controversial. In agreement with the current findings, the Cardiovascular Health Study $(n=5,201)$ [16] found an independent inverse relationship between FEV1 and AF with an RR of 0.75 (confidence interval $0.59-0.94$ ) per measured litre of FEV1 during a relatively short-term follow-up period of 4 yrs. In contrast, the Renfrew/Paisley Study $(n=15,406)$ [17] did not demonstrate any significant correlation between FEV1 level and AF. Similarly, the Framingham Study ( $n=4,731)$ [13] found no relationship between FEV1 \% pred and AF in biennial investigations during 38 yrs of follow-up.

The Renfrew/Paisley Study only observed a small number of cases with new AF $(n=19)$ after a short follow-up, and hence, only the strongest predictors would be significant. They also compared FEV1 and other variables with incident hospital admission for AF during 20 yrs. This follow-up is long compared with the present study and it is likely that this could have affected the results. For instance, the risk of cardiomegaly, which was a very strong predictor at shorter follow-up, was reduced nearly 10 times at longer follow-up. In the Framingham Study, there was a high rate of heart disease. For instance, the prevalence of valvular heart disease was $7 \%$ for males and $9 \%$ for females. Therefore, their population was different from the present one and probably not quite comparable. Furthermore, new cases of AF in the Framingham Study included recurrent episodes of AF, which could also have contributed to the different results because predictors of new onset $\mathrm{AF}$ and recurrence of AF need not be the same.

The present results could not entirely be explained by smoking. The relationship between smoking and risk of AF was only present in the heaviest smoking group at hospitalisations but not at re-examination. None of the abovementioned prospective studies have found smoking to be an independent predictor. Besides, the smoking rate of participants in the Renfrew/Paisley study was similar to the smoking rate in the CCHS. The authors did not have any methods to assess the prevalence of valvular disease or congestive heart failure in this population. It could be argued that subjects with heart failure have some reduction in lung function that could explain the relationship between FEV1 and AF in the CCHS. Against this argument is the fact that the Cardiovascular Health Study included both ejection fraction and atrial size in their analyses and reached similar results to the present study. The present study found that $<2 \%$ of hospital diagnoses could be attributed to valvular heart disease when investigating main diagnoses for hospitalisations associated with AF as second discharge diagnosis. Therefore, valvular heart disease was assumed to be a rare cause of AF in this population. An association between AF and use of diuretics was not found, although use of diuretics is an imprecise measurement of chronic heart failure. Still, it would probably include most cases of symptomatic heart failure in the study population because angiotensin-converting enzyme inhibitors were not commonly used drugs at the time of data collections. Unfortunately there was no available data on asthma medications at baseline. The literature on $\beta_{2}$-agonist-induced AF is sparse and contains only casuistic reports. In general, inhaled sympathomimetics, studied extensively for treatment of asthma, have been found to be safe from cardiac arrhythmias [19]. It is thus unlikely that this would have confounded the results.

AF detected at examinations would include all cases of chronic AF (or frequently recurrent cases), whereas using hospital discharge records would tend to identify subjects with more symptomatic AF. Still, the results were similar regardless of whether AF was detected at examination or at 
hospitalisation. Using all AF hospitalisations as end-points could cause a selection bias, since subjects admitted for COPD, for example, would be more likely to have concurrent AF diagnosed. However, only $\sim 3 \%$ of admissions with AF as a secondary diagnosis were primarily classified as COPD or asthma with a further $10 \%$ of diagnoses due to pneumonia, which could include undiagnosed COPD admissions. In addition, results were similar whether AF was the main or a secondary discharge diagnosis. As with other epidemiological studies, nonresponse could have caused bias if nonresponse were associated with both poor lung function and risk of AF, which there is no reason to assume.

The mechanism connecting reduced lung function with AF is not clear. Recent observations have revealed that ectopic beats initiating AF often originate in the walls of the pulmonary veins [20], and it is possible that this could be triggered by changes in gas composition or pulmonary hypertension. In the present study, hypoxia and cor pulmonale could only account for some of this effect since the relationship was also found in subjects with mild to moderately reduced FEV1. Reduced lung function has been shown to be an independent predictor of IHD [21-23] and of stroke [24, 25], and it is possible that the biological mechanism for development of AF could be linked to atherosclerosis via a common pathway of the development of vascular and airways disease, e.g. foetal or early childhood exposure.

In conclusion, this study indicates that reduced forced expiratory volume in one second \% predicted is an independent predictor of new onset atrial fibrillation. Since atrial fibrillation, if untreated, causes high morbidity from stroke and is associated with increased mortality [26], this indicates the importance of routine electrocardiograms in patients with chronic obstructive pulmonary disease. This study was not designed to detect causal mechanisms and further investigations are needed to clarify the biological pathways that connect reduced lung function with atrial fibrillation.

\section{References}

1. Khokhar N. Cardiac arrhythmias associated with acute respiratory failure in chronic obstructive pulmonary disease. Milit Med 1981; 146: 856-858.

2. Sideris DA, Katsadoros DP, Valianos G, Assioura A. Type of cardiac dysrhythmias in respiratory failure. Am Heart $J$ 1975; 89: 32-35.

3. Levine PA, Klein MD. Mechanisms of arrhythmias in chronic obstructive lung disease. Geriatrics 1976; 31: 47-56.

4. Thomas AJ, Valabhji P. Arrhythmia and tachycardia in pulmonary heart disease. Br Heart J 1969; 31: 491-495.

5. Holford FD, Mithoefer JC. Cardiac arrhythmias in hospitalized patients with chronic obstructive pulmonary disease. Am Rev Respir Dis 1973; 108: 879-885.

6. Shih HT, Webb CR, Conway WA, Peterson E, Tilley B, Goldstein S. Frequency and significance of cardiac arrhythmias in chronic obstructive pulmonary disease. Chest 1988; 94: $44-48$.

7. Hurd S. The Impact of COPD on Lung Health Worldwide. Chest 2000; 117: 1S-4S.
8. Murray CJL, Lopez AD. Evidence-based health policy Lessons from the global Burden of Disease Study. Science 1996; 274: 740-743.

9. Appleyard M, Hansen AT, Schnohr P, Jensen F, Nyboe J. The Copenhagen City Heart Study: A book of tables with data from the first examination (1976-78) and a 5-year follow-up (1981-83). Scand J Soc Med 1989; 170: 1160.

10. Rose GA, Blackburn H. Cardiovascular survey methods. Monograph Series. Geneva, World Health Organization, 1968; p. 56.

11. Berkson J. Limitations of the application of fourfold table analysis to hospital data. Biomet Bull 1946; 2: 47-53.

12. Lange P. Development and prognosis of chronic obstructive pulmonary disease with special reference to the role of tobacco smoking. Dan Med Bull 1992; 39: 30-48.

13. Benjamin EJ, Levy D, Vaziri SM, Dágostino RB, Belanger AJ, Wolf PA. Independent risk factors for atrial fibrillation in a population-based cohort: the Framingham Heart Study. JAMA 1994; 271: 840-844.

14. Krahn AD, Manfreda J, Tate RB, Mathewson FAL, Cuddy TE. The natural history of atrial fibrillation: incidence, risk factors, and prognosis in the Manitoba Follow-Up Study. Am J Med 1995; 98: 476- 484.

15. Furberg CD, Psaty BM, Manolio TA, Gardin JM, Smith VE, Rautaharju PM. Prevalence of atrial fibrillation in elderly subjects (the Cardiovascular Health Study). Am J Cardiol 1994; 74: 236-241.

16. Psaty BM, Manolio TA, Kuller LH, et al. Incidence of and risk factors for atrial fibrillation in older adults. Circulation 1997; 96: 22455-22461.

17. Stewart S, Hart CL, Hole DJ, McMurray JJ. Population prevalence, incidence, and predictors of atrial fibrillation in the Renfrew/Paisley study. Heart 2001; 86: 516-521.

18. Cox DR. Regression modes and lifetables. J R Stat Soc 1972; B34: 187-220.

19. Breeden CC, Safirstein BH. Albutarol and spacer-induced atrial fibrillation. Chest 1990; 98: 762-763.

20. Olsson SB. Atrial fibrillation - Where do we stand today? J Intern Med 2001; 250: 19-28.

21. Kannel WB, Hubert H, Lew EA. Vital capacity as a predictor of cardiovascular disease: the Framingham Study. Am Heart J 1983; 105: 311-315.

22. Persson C, Bengtsson C, Lapidus L, Rybo E, Thiringer G, Wedel H. Peak expiratory flow rate and risk of cardiovascular disease and death. A 12 year follow-up of participants in the population study of women in Gothenburg, Sweden. Am J Epidemiol 1986; 124: 942-948.

23. Lange P, Nyboe J, Jensen G, Schnohr P, Appleyard M. Ventilatory function impairment and risk of cardiovascular death and of fatal or non-fatal myocardial infarction. Eur Respir J 1991; 4: 1080-1087.

24. Truelsen T, Prescott E, Lange P, Schnohr P, Boysen G. Lung function and risk of fatal and non-fatal stroke. The Copenhagen City Heart Study. Int J Epidemiol 2001; 30: $145-151$.

25. Wannamethee G, Shaper AG, Ebrahim S. Respiratory function and risk of stroke. Stroke 1995; 26: 2004-2010.

26. Lake FR, Cullen KJ, de Klerk NH, McCall MG, Rosman DL. Atrial fibrillation and mortality in an elderly population. Aust N Z Med 1989; 19: 321-326. 